Mathématiques et sciences humaines
Mathematics and social sciences

170 | Printemps 2005

Mathématiques, jeux sportifs, sociologie

\title{
Essai sur les sports de course
}

Trials on race sports

Luc Collard

\section{(2) OpenEdition}

Journals

Electronic version

URL: http://journals.openedition.org/msh/2948

DOI: $10.4000 / \mathrm{msh} .2948$

ISSN: 1950-6821

\section{Publisher}

Centre d'analyse et de mathématique sociales de l'EHESS

\section{Printed version}

Date of publication: 1 March 2005

ISSN: 0987-6936

\section{Electronic reference}

Luc Collard, «Essai sur les sports de course », Mathématiques et sciences humaines [Online], 170 | Printemps 2005, Online since 05 April 2006, connection on 23 July 2020. URL : http:// journals.openedition.org/msh/2948; DOI : https://doi.org/10.4000/msh.2948 


\title{
ESSAI SUR LES SPORTS DE COURSE
}

\author{
Luc COLLARD ${ }^{1}$
}

RÉSUMÉ - Les sports de course à structure de coalitions de joueurs (moto-cross, cyclisme sur route, etc.) font tache dans l'univers des sports car ce sont les seuls où les relations interpersonnelles peuvent être ambivalentes. En témoigne une analyse à l'aide de la Théorie des jeux des stratégies à trois joueurs. La réussite du pilote peut cependant profiter d'interactions de coopération exclusive, mais à condition que canal et codes de communication soient maîtrisés ; ce qui ne semble pas évident au regard des réponses bigarrées de quelques dizaines de motards interrogés. L'analyse mathématique milite en faveur d'un entraînement fondé sur une sémiologie de la motricité.

MOTS-CLÉS - Sémiologie, Sport de course, Théorie des jeux

SUMMARY - Trials on race sports

Race sports with a player-coalition structure (motorcycle scramble, cycling on roads...) are a stain in the world of sports because they are the only ones in which interpersonal relations can be ambivalent. To testify this: here is an analysis through the 3-player strategy games theory. The solution of theses different situations can take advantage of the exclusive cooperative interplay but only if ways and codes of communications are mastered; which is far from being obvious when we have a look at the multifaceted answers of a dozen of motorcyclists who were interviewed. The mathematical analysis is fighting for a training based on semiology of motor functions.

KEY-WORDS - Race sports, Semiology, Theory of games

\section{INTRODUCTION. UNE APPARITION RÉCENTE}

Apparemment quoi de plus naturel et d'universel que les sports de course!? Défier des concurrents sur une distance à parcourir en un minimum de temps, à pied sec ou dans l'eau, à cheval, en vélo, à moto, ou à roller, voilà qui semble bien à la base d'une myriade de jeux sportifs. N'est-ce pas là un des traits de logique motrice les plus évidents!?

En réalité, à partir d'un corpus de 150 jeux peints et commentés par P. Bruegel (1560) et J. Stella (1657), P. Parlebas [2003] ne décrypte pas la moindre trace de confrontation de type course entre individus ou entre équipes à la Renaissance!! L'époque fourmille pourtant de jeux physiques originaux (un contre tous, une équipe contre toutes les autres, chacun pour soi, etc.) qui, comme certains sports de course moderne (courses de moto-cross, courses cyclistes, courses de char à voile, etc.) peuvent être rangés dans les «!jeux à $n$ joueurs et à somme non nulle »!: dans ce type de jeu, le

\footnotetext{
${ }^{1}$ Groupe d'Etude Pour l'Europe de la Culture et de la Solidarité GEPECS (Axe 5), Université Paris 5 12, rue Cujas 75230 Paris cedex 05, luc.collard@u-picardie.fr
} 
gain des vainqueurs n'est pas égal à la perte des vaincus. Ces jeux se distinguent de ceux que J. Von Neumann [1944] appelle les «!jeux à 2 personnes et à somme nulle », plus souvent désignés comme des «!duels!» [1968], autrement dit des jeux strictement compétitifs où tout ce qu'un joueur gagne, l'autre le perd (jeux de paume, football, sports de combat, etc.).

Si les duels ludomoteurs sont déjà bien présents à la Renaissance - pour environ 30 à $40 \%$ des jeux de l'époque (la tendance se confirme aujourd'hui) - les jeux de course y sont donc curieusement absents. En fait, ils apparaissent quand naît le sport «!sport!» étant pris ici dans son acception forte - au XVIII ${ }^{\mathrm{e}}$ siècle en Grande-Bretagne et un peu plus tard en France. Dans cette période et jusqu'au début du $\mathrm{XX}^{\mathrm{e}}$ siècle en France, sur une quarantaine de sports présents (d'abord organisés sur le mode associatif par des clubs, puis régis par des fédérations), douze, soit environ 1/3 du total, sont des courses ou possèdent des épreuves de courses!poursuites ou au coude à coude :

- hippisme (1776),

- aviron (1830),

- yachting (1851),

- courses de natation (1880),

- courses cyclistes (1881),

- courses d'athlétisme (1882),
- courses automobiles (1895),

- char à voile (1896),

- motocyclisme (1904),

- épreuves de pentathlon (1906),

- patinage de vitesse (1908),

- motonautisme (1912).

Cette fulgurante apparition est révélatrice des bouleversements socioéconomiques et culturels de l'époque. Ces sports représentent les passe-temps à la mode, aux normes des nouveaux riches de l'ère industrielle!: rentiers, chefs d'entreprises, riches commerçants. Ces derniers sont désormais les seuls à pouvoir s'accorder des plages de loisir. Ce sont les maîtres du temps et semblent bien vouloir le vérifier sur le plan ludique en affrontant leurs pairs à travers des courses où, seul, compte le chronomètre. Et puis, le XVIII et $\mathrm{XX}^{\mathrm{e}}$ siècle, c'est aussi l'eldorado de l'inventivité technique enfin débarrassée des interdits religieux. Les sports de course vont abondamment puiser dans les nouvelles technologies (avant même de les impulser)!: vélo, moteur, voile, etc. La rentabilité est le maître-mot, y compris dans les sports de course n'utilisant pas d'agent de locomotricité externe comme la natation ou l'athlétisme. Ici, on copie la machine et on innove à tout prix. En natation, par exemple, pas moins de six techniques de nage verront le jour à cette période!; deux de ces six leur survivront dans le sport d'aujourd'hui.

Face à l'effervescence de ces nouvelles techniques un problème se fera jour!: comment assurer l'égalité des chances!? Comment être certain que les protagonistes ne triomphent que par leurs mérites intrinsèques et non par les services d'une technologie plus heureuse!? La «!sportification!» des pratiques corporelles va de pair avec l'uniformisation des droits et interdits relative à l'accomplissement moteur. A leur naissance, les sports de course opposaient les styles et les techniques!; c'est l'ingéniosité des joueurs et des ingénieurs qui faisaient le sel du jeu!: on gagnait grâce à des savoirfaire originaux (comme en natation) à l'utilisation d'instruments techniques novateurs (moteurs suralimentés, freins à disques, bras oscillants indépendants, etc., en course automobile ou en motocyclisme). Comment cela était-il compatible avec la standardisation sportive!? La tendance majeure fut la mise en conformité réglementaire, en statuant sur les rapports à autrui, à l'espace, aux instruments techniques. Par voie de conséquence, toute action motrice innovante, tout engin médiateur non conforme, seraient désignés, débattus et parfois combattus. Les nouveaux sports de course apparus 
depuis (triathlon, courses de rollers, de planches à voile, de cyclo-cross, de side-car cross, de motoneiges, etc.) ont dû tous se plier à cette campagne d'uniformisation.

Nés sous le signe de l'inventivité et de la diversité associées aux développements techniques et scientifiques les plus en pointe, les jeux de course ont été mis au pas à travers leur sportification. Étant dépossédés de leur fonction originaire, que sont-ils devenus aujourd'hui!(cf. §1.) ? Peut-on encore tous les classer comme des «!sports à $n$ joueurs et à somme non nulle!»!(cf. §2.) ? Nous traiterons de ces questions en confrontant quelques données de la théorie des jeux aux spécificités des actions motrices engagées (cf. § 3., 4. et 5.)!: comment fonctionne cette catégorie de sports!? Nous verrons également que les opinions des spécialistes de ces pratiques sont révélatrices d'une vision partielle de ce qui s'y joue ou peut s'y jouer (cf. §6.). La mise au jour des caractéristiques mathématiques originales des sports de course nous permettra, au final, d'avancer l'hypothèse selon laquelle la logique sportive majoritairement adoptée par les pilotes tend fondamentalement à s'opposer au principe même de ces jeux.

Qui sont donc ces trublions du sport et comment l'Institution parvient-elle à les dompter!?

\section{DE LA DIFFERENCIATION DANS LES SPORTS DE COURSE}

Il y a bien sûr les descentes, les slaloms géants du ski, les épreuves contre la montre individuelles en cyclisme qui font intervenir les joueurs un par un. Toutefois, dans l'imaginaire collectif, les épreuves reines des courses - comme la finale du 100 mètres aux Jeux olympiques!- sont associées aux oppositions interindividuelles. Le 16 juin 2000 , le célèbre nageur russe, A. Popov, complètement remis du coup de couteau qui faillit lui coûter la vie quinze jours après son retour victorieux des Jeux olympiques d'Atlanta (1996), allait pourtant irréfutablement apporter la preuve que tous les sports de course au coude à coude ne sont pas à $n$ joueurs. Lors des épreuves officielles de sélection pour les Jeux de Sydney, il rate son épreuve de 50 mètres nage libre à la suite d'un faux départ. S'adressant au responsable de la compétition, il écrit dans son livre autobiographique [Popov, 2001]!:

Ce faux départ a quelque peu faussé ma course. J'aimerais bien faire une autre tentative en solitaire à la ligne d'eau $n^{\circ} 4$ (p. 154).

Après un instant d'hésitation, le juge arbitre souligne qu'en rajoutant une autre épreuve à la compétition, d'autres nageurs voudront fort logiquement y participer.

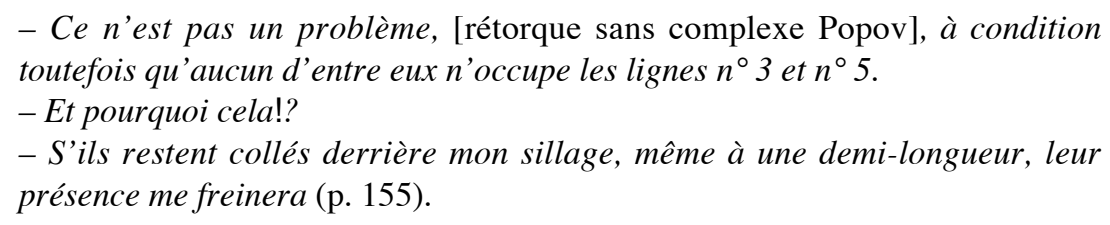

Ayant plié aux exigences du Tsar russe, les Officiels durent accepter le lendemain les 21 secondes 64 centièmes de l'affichage électronique consacrant Popov comme le nageur le plus rapide du monde sur cette distance en grand bassin. Ce record est inégalé à ce jour (05-05-2005). D'ailleurs, les images vidéo sous-marines de son plus récent triomphe aux Championnats du monde à Barcelone en 2003 signalent que le «!Tsar!» applique ce qu'il écrit!: on le voit se forcer à tendre la nuque et à orienter son regard vers le fond de la piscine au moment du sprint final... En somme, pour réussir à ce niveau, il semble 
qu'il faille, dans certains cas, renoncer ni plus ni moins à prélever de l'information sur ce que sont en train de faire les concurrents. Puisqu'il s'agit de situation «!psychomotrice!», ne porter aucune attention aux adversaires est une option rentable. Inutile de préciser qu'un tel comportement en boxe ou en rugby - activités «!sociomotrices!»- serait absurde.

Voici la preuve, s'il en était encore besoin, que les courses en couloirs de natation (mais aussi d'athlétisme!ou d'aviron, sans partenaire) sont exemptes d'interaction motrice!; et que les relations psychoaffectives qui y ont cours ne sont que des éléments perturbateurs d'une prestation physique individuelle. Ces sports sont devenus de pure habileté!; il s'agit d'une juxtaposition de stéréotypes en solo, soigneusement réglés à l'entraînement. On est loin des premières courses de natation en rivière, sans couloir, où prendre une demi-longueur à un adversaire pouvait permettre «!en force!» de l'empêcher de passer. En triathlon, par exemple, il est désormais interdit aux cyclistes de se suivre roue dans roue, pour ne pas que le résultat final trahisse une association de fortune. Il faut que les prestations individuelles de chacun soient comparables dans l'absolu à celles des autres et les organisateurs cherchent coûte que coûte à museler les conduites d'interactions qui pourraient donner crédit à des relations perfides.

Les sports de course arborent désormais plusieurs facettes. Il y a ceux qui sont «!psychomoteurs!», en solo (ski alpin) ou au coude à coude sans interaction (courses individuelles en couloir en athlétisme)!; ils sont à ranger dans la catégorie des jeux à un joueur et n'ont de course que le nom (cf. Figure 1). De même pour les courses de stricte coopération motrice (relais en couloir, aviron) où chaque équipe, en co-motricité avec les autres, est en fait un «!super joueur ». Les intérêts de joueurs intra-équipes y sont totalement solidaires et leur résolution ne pose guère de problème en terme décisionnel!: dans les relais de natation, par exemple, en faisant l'addition des quatre meilleurs temps de chaque concurrent on peut prédire quasiment à tous les coups le résultat final, avant même de savoir qui nagera en premier ou en second...

\begin{tabular}{|l|l|l|}
\hline \multicolumn{1}{|c|}{ Type de courses } & Nature des relations & \multicolumn{1}{c|}{ Illustrations } \\
\hline \multirow{2}{*}{$\begin{array}{l}\text { Courses } \\
\text { sociomotrices } \\
\text { (présence d'interaction } \\
\text { motrice) }\end{array}$} & $\begin{array}{l}\text { Coalition de } \\
\text { joueurs }\end{array}$ & $\begin{array}{l}\text { Moto-cross, ski de fond, courses de demi-fond, Formule } \\
\text { 1, etc. }\end{array}$ \\
\cline { 2 - 3 } & Coalition d'équipes & Régates par équipes, relais de demi-fond, etc. \\
\cline { 2 - 3 } $\begin{array}{l}\text { Courses } \\
\text { psychomotrices } \\
\text { (absence d'interaction } \\
\text { motrice) }\end{array}$ & Stricte coopération & Courses d'aviron, relais de natation sportive, etc. \\
\cline { 2 - 3 } & En solo & $\begin{array}{l}\text { Courses en couloirs (athlétisme, natation sportive), } \\
\text { épreuves parallèles en ski, etc. }\end{array}$ \\
\hline
\end{tabular}

Figure 1. Distribution des sports de course selon la présence ou l'absence d'interaction motrice

Contrairement à une idée reçue, l'absence d'adversaire direct est fréquente dans ces sports y compris lorsque les protagonistes sont au coude à coude (comotricité) comme dans les épreuves individuelles de natation sportive.

Il reste ensuite les courses «!sociomotrices!» sous forme de coalitions de $n$ joueurs ou de $n$ super joueurs autrement dit!sous forme d'équipes [P. Parlebas, 2002]. Ce sont les courses dont la structure est la plus proche de l'esprit originaire (courses hippiques, premières courses d'athlétisme ou de natation, etc.). Les prises de décision liées aux interactions essentielles fondent la stratégie praxique qui peut faire l'objet d'une analyse mathématique, ainsi que nous nous y essayerons aux points 4 . et 5 . 
Désormais, lorsque nous évoquerons les sports de course nous parlerons exclusivement de ces situations de coalitions de joueurs (moto-cross, cyclisme sur route, Formule 1, etc.). Notons que, dans le domaine des pratiques motrices institutionnalisées, elles sont désormais peu typiques!: on peut estimer qu'elles ne regroupent que $6 \%$ de l'effectif total des sportifs aujourd'hui.

\section{DES JEUX SEMI-COOPERATIFS A INFORMATION INCOMPLETE}

C'est sans doute sur le versant de l'information dont les protagonistes bénéficient et sur la nature des relations qui unissent ceux-ci, que les sports de course se démarquent le plus des autres sports sociomoteurs.

Les courses sociomotrices sont les seuls sports continus à actions simultanées où les joueurs disposent d'informations partielles sur ce que les autres font et sont capables de faire, compte tenu de leur état. L'introduction de points aveugles dans l'information et la présence de plusieurs joueurs $(n>2)$ limitent les participants dans leur connaissance du domaine de choix et du type de comportement de tous. Ce sont des jeux à information incomplète. En cela, ils se distinguent fondamentalement des duels et des sports psychomoteurs (gymnastique, lancer de poids, haltérophilie) qui sont des jeux à information complète. Par contre, pour les courses comme pour les autres sports, les issues du jeu et les gains qui leur sont associés sont connus de tous.

Dans ces sports à information incomplète chacun est informé de son état avant de jouer. Un coureur n'est pas informé de l'état des autres coureurs mais sait qu'ils le sont de leur propre état. C'est le cas le plus typique des courses!: l'information est privée. Je ne sais pas si mes adversaires devront ravitailler avant la ligne d'arrivée mais je sais qu'ils le savent. Plus rarement, il arrive aussi que le joueur ne soit pas informé de l'état des autres coureurs et qu'il ne sache pas non plus que les autres le sont. L'information est alors secrète. Je ne sais pas si mes adversaires devront ravitailler avant la ligne d'arrivée et je ne sais pas qu'ils le savent. Ce qui importe dans l'analyse de ce type de situation ce n'est pas tant l'incertitude elle-même (devront-ils oui ou non ravitailler!?) mais les différences d'information sur cette incertitude (je sais ou je ne sais pas qu'ils savent s'ils doivent ravitailler). La prise en compte des différents «!états!», éventuellement sous couvert de probabilités, est un élément-clef de la résolution de ces jeux.

Mais les relations que peuvent entretenir les joueurs entre eux viennent vite compliquer l'affaire. Les intérêts des coureurs ne sont pas nécessairement antagonistes et peuvent présenter de réelles convergences. C'est précisément en cela que les courses, contrairement aux duels, sont dites à somme non nulle. Deux adversaires peuvent provisoirement coopérer pour battre un troisième avant d'en découdre entre eux. Deux partenaires peuvent subitement devenir adversaires à l'approche de la ligne d'arrivée. De tels actes sont impensables en football ou en basket-ball où ils seraient jugés frauduleux et scandaleux. Les jeux semi-coopératifs laissent la porte ouverte aux connivences fortuites, aux arrangements prémédités. Les intérêts interpersonnels ne sont pas diamétralement opposés.

Il est à noter qu'en sport, seules les courses à $n$ joueurs peuvent être qualifiées de jeux semi-coopératifs (cf. Figure 2). Voilà qui n'est pas sans poser problème sur le plan de l'éthique sportive. Et si les pilotes d'une même écurie dominante concluaient un marché pour gagner les Grands prix à tour de rôle!? Et si deux coureurs de demi-fond se 
partageaient les victoires sur 800 et 1500 mètres!? Le bruit courut en effet en juillet 1980, à Moscou. Le demi-fondeur anglais Steve Ovett, incontesté sur $1500 \mathrm{~m}$ - il avait quand même remporté quarante-six courses consécutivement sur cette distance!! - prit curieusement la seconde place en finale des Jeux olympiques derrière son compatriote, le malheureux Sebastian Coe. Malheureux car, victorieux de ses vingt-six derniers 800 mètres, Coe, grand favori sur cette distance, avait lui-même été battu six jours plus tôt par... Steve Ovett. Un chassé-croisé que beaucoup considérèrent comme trop proche du conte de fée. De telles suspicions, fondées ou pas, ne peuvent naître que de sports laissant prise à l'ambivalence. Et c'est bien là, à la fois le talon d'Achille et la pierre angulaire des sports de course...

\begin{tabular}{|l|l|}
\hline \multicolumn{1}{|c|}{$\begin{array}{c}\text { Informations } \\
\text { sur les } \\
\text { concurrents }\end{array}$} & \multicolumn{1}{|c|}{ Illustrations } \\
\hline $\begin{array}{l}\text { Jeu à } \\
\text { information } \\
\text { complète }\end{array}$ & $\begin{array}{l}\text { Football, tennis, judo, } \\
\text { volley-ball, etc. }\end{array}$ \\
\hline $\begin{array}{l}\text { Jeu à } \\
\text { information } \\
\text { incomplète }\end{array}$ & $\begin{array}{l}\text { Cyclisme sur route, moto- } \\
\text { cross, demi-fond, Formule } \\
1, \text { etc. }\end{array}$ \\
\hline $\begin{array}{l}\text { Jeu sans } \\
\text { information }\end{array}$ & $\begin{array}{l}\text { Rallye enduro type Paris- } \\
\text { Dakar (parfois) }\end{array}$ \\
\hline
\end{tabular}

\begin{tabular}{|l|l|}
\hline $\begin{array}{l}\text { Relations entre } \\
\text { les concurrents }\end{array}$ & \multicolumn{1}{|c|}{ Illustrations } \\
\hline Jeu coopératif & $\begin{array}{l}\text { Patinage mixte, aviron (à } \\
\text { plusieurs rameurs), etc. }\end{array}$ \\
\hline $\begin{array}{l}\text { Jeu semi- } \\
\text { Coopératif }\end{array}$ & $\begin{array}{l}\text { Cyclisme sur route, moto- } \\
\text { cross, demi-fond, Formule } \\
\text { 1, etc. }\end{array}$ \\
\hline $\begin{array}{l}\text { Jeu strictement } \\
\text { Compétitif }\end{array}$ & $\begin{array}{l}\text { Football, tennis, judo, } \\
\text { volley-ball, etc. }\end{array}$ \\
\hline
\end{tabular}

Figure 2. Comparaison des courses avec interaction motrice aux autres sports sociomoteurs selon le degré d'information et la nature des relations entre les joueurs Les courses sont les seuls sports sociomoteurs semi-coopératifs à information incomplète (et dans de très rares cas sans information).

\section{DEUX TYPES D'INTERACTION MOTRICE}

À nouveau nous nous référons aux concepts pionniers de praxéologie motrice avancés par P. Parlebas [1999]. Dans tout sport de course sociomoteur il peut y avoir deux types d'interaction!: «!'interaction motrice directe!» d'une part, faite de contrecommunications comme bloquer, empêcher de passer, bousculer, etc. (en moto-cross, par exemple) et de communications motrices comme aider, assurer, etc. (en régates de voile, par exemple)!; et «!l'interaction motrice indirecte!» d'autre part, faite de «!praxèmes!» et de «!gestèmes!».

Un praxème est la conduite motrice d'un joueur interprétée comme un signe dont le signifiant est le comportement observable et le signifié le projet tactique correspondant tel qu'il a été perçu (p. 260). Il s'agit de comportements apparemment anodins qui correspondent en réalité à des actes de préaction - comme se déporter à l'extérieur de la piste, retarder son freinage en entrée de virage, etc. (en course automobile, par exemple) - porteur de messages devant être, si possible, transparents pour les alliés et troubles pour les ennemis.

Un gestème est la classe d'attitudes, de mimiques, de gestes, de comportements moteurs accomplis dans le but de transmettre soit une demande, soit une indication, soit une injonction tactique ou relationnelle par simple substitution à la parole! (p. 155)!: il peut s'agir d'un mouvement de bras, d'un hochement de tête, annonciateur d'une action de coopération (courses de relais en athlétisme lors du passage de témoin, par exemple) ou d'opposition (menace d'un cycliste en réaction au refus d'un autre de prendre le relais en tête d'échappée, par exemple). Praxèmes et gestèmes nécessitent des facultés 
de décentration et d'anticipation de haute volée et l'acquisition d'un code spécifique!à chaque sport sociomoteur (moto-cross, escrime, volley-ball, etc.) : tout joueur doit prendre conscience des processus d'encodage et de décodage nécessaires à l'interaction. L'assimilation de ce type d'interaction indirecte est bien sûr au service de la performance sportive!; mais elle n'en est pas moins un élément éducatif intéressant et en grande partie la source du plaisir du jeu.

L'interaction motrice directe, quant à elle, possède une particularité dans les sports de course comparée à celle rencontrée dans les duels sportifs. En football, en tennis ou en judo - les archétypes des duels européens - pour gagner il faut contrecommuniquer!: tirer, frapper, plaquer. La marque n'évolue que par actes d'interactions directes. Il en va différemment dans les courses où les contre-communications peuvent affecter tout autant les agresseurs que les agressés, et ne rien apporter au classement final. Lorsque deux pilotes de Formule 1 se tamponnent, il est rare que l'un d'eux en réchappe. On voit bien que la logique est différente en boxe où l'objectif est précisément de se «!tamponner!». D'ailleurs il arrive que le code du jeu interdise les contre-communications, comme en cyclisme sur route, afin d'éviter les règlements de compte ou les sacrifices individuels pour l'équipe!; empêcher un adversaire de doubler en sprint, le coincer dans les barrières, le retenir par le maillot dans le peloton peuvent entraîner sanctions et disqualifications. Cela n'empêche pas les démarrages au bluff, les ralentissements forcés ou les blocs de rassemblement d'équipiers en tête mais qu'il faut interpréter ici comme des praxèmes, c'est-à-dire des unités tactiques à part entière (cf. Figure 3).

\begin{tabular}{|c|c|c|c|}
\hline $\begin{array}{c}\text { Type } \\
\text { d'interaction }\end{array}$ & $\begin{array}{c}\text { Type de } \\
\text { communication }\end{array}$ & Illustration en moto-cross & $\begin{array}{c}\text { Illustration en cyclisme sur } \\
\text { route }\end{array}$ \\
\hline \multirow{2}{*}{$\begin{array}{l}\text { Interaction } \\
\text { motrice } \\
\text { directe }\end{array}$} & $\begin{array}{l}\text { Communication } \\
\text { motrice }\end{array}$ & $\begin{array}{l}\text { S'arrêter pour aider un pilote à } \\
\text { relever sa moto après une chute } \\
\text { (du jamais vu, sauf en Enduro) }\end{array}$ & $\begin{array}{l}\text { Aider en le poussant un } \\
\text { coureur à la traîne (parfois } \\
\text { interdit) ... }\end{array}$ \\
\hline & $\begin{array}{l}\text { Contre- } \\
\text { communication } \\
\text { motrice }\end{array}$ & $\begin{array}{l}\text { Bousculer (du nom originel du } \\
\text { moto-cross : scramble), faire } \\
\text { chuter un adversaire... }\end{array}$ & $\begin{array}{l}\text { Bloquer un coureur dans le } \\
\text { peloton (théoriquement } \\
\text { interdit)... }\end{array}$ \\
\hline \multirow{2}{*}{$\begin{array}{l}\text { Interaction } \\
\text { motrice } \\
\text { indirecte }\end{array}$} & Praxème & $\begin{array}{l}\text { Décrocher à l'extérieur et } \\
\text { doubler... }\end{array}$ & $\begin{array}{l}\text { Une échappée au pied d'un } \\
\text { col... }\end{array}$ \\
\hline & Gestème & $\begin{array}{l}\text { Mouvement de tête destiné à } \\
\text { transmettre une information... }\end{array}$ & $\begin{array}{l}\text { Signe de la main ou cri } \\
\text { annonçant la mise en place } \\
\text { d'une tactique... }\end{array}$ \\
\hline
\end{tabular}

Figure 3. Façons de communiquer dans les sports de course L'interaction motrice directe n'est pas privilégiée dans ce type de situation y compris pour le moto-cross où bousculer peut affecter tout autant l'émetteur que le récepteur. La logique est bien différente dans le cas des duels où elle constitue le corps du jeu.

D'un point de vue médiatique, hélas, l'interaction motrice indirecte n'est pas aussi accessible au grand public que les interactions de marque. Un smash ou un penalty est lisible par tous!; un ralentissement brusque ou une amorce de démarrage l'est beaucoup moins. Les courses à $n$ joueurs ne se donnent pas aussi facilement en spectacle que les duels. Par contre, à l'instar des duels d'équipe (rugby, hand-ball, etc.), les communications motrices des courses peuvent être au service d'une équipe comme en side-car, en régate et même d'une certaine façon en course cycliste par équipes (prendre le relais en cyclisme n'est pas seulement un acte tactique, un praxème, c'est pour des raisons aérodynamiques avérées une façon d'économiser les forces des «!suceurs de roue!»). 
Rentrons à présent plus précisément dans ce qui se joue dans ces jeux!; en partant du plus simple!: les relations d'antagonisme entre deux coureurs.

\section{RESOLUTION DES OPPOSITIONS DUELLES}

Contrairement à sa dénomination originelle, la bousculade n'est pas l'interaction la plus recherchée du moto-cross. Engagés de front, «!poignées en coin!» avec des motos de même cylindrée mais de performances distinctes, par rangées de $20,30,40, \ldots$, tout droit vers l'entonnoir du premier virage, la plupart des concurrents ne peuvent échapper au «!scramble!». Aucun d'entre eux ne souhaite l'impact. Il ne profite qu'à ceux qui en réchappent. C'est plutôt dans les interactions de type tactique que se révèlent les principes d'action du moto-cross, du cyclisme et plus globalement des sports de course de type sociomoteur. Les «!praxèmes!» correspondent aux conduites de pilotage interprétées comme des signes par les protagonistes. Chaque signe comporte un signifiant $(\mathrm{Sa}$ ) - qui est le comportement observable - et un signifié (Sé) - qui est l'intention tactique telle qu'elle a été perçue. Un praxème peut être interprété à plusieurs niveaux. Prenons, pour commencer, le cas des interactions de deux pilotes, J1 et J2, à l'entrée d'un virage. C'est l'un des moments - avec les sauts - les plus propices au doublement.

Le premier niveau correspond au jugement des profanes, à la dénotation évidente des actions engagées. D'un point de vue purement biomécanique, le freinage de J1 et J2 à l'extérieur et avant le virage (Sa1) annonce une accélération des deux pilotes pendant (Sé1). Mais, à vrai dire, ce que voient les pilotes experts sur le vif est un peu plus affûté. Ce premier signe - freinage annonçant une accélération - devient le signifiant d'un autre signe, un signe de connotation. Pour le devançant, il peut s'agir d'un positionnement d'anticipation destiné à tracer une trajectoire intérieure courte (J1-Sa2) afin de tenter de maintenir son avance (J1-Sé2)!; pour le devancé, ce même niveau de tactique franche le conduirait également au plus court chemin (J2-Sa2) dont le signifié serait la tentative de doublement à l'intérieur (J2-Sé2). Cette représentation anticipée de la situation peut se réitérer à un autre degré, sur le plan de la ruse. Ainsi, le devancé, qui pourrait feinter en attaquant à l'extérieur $(\mathrm{J} 2-\mathrm{Sa} 3)$ tentant ainsi un dépassement là où on ne l'attend pas (J2-Sé3), peut très bien inspirer le devançant qui, restant également à l'extérieur (J1-Sa3) pourrait lui fermer la porte (J1-Sé3)...

Mais, que se passerait-il si la tactique franche de l'un rencontrait la ruse de l'autre!? Quel profit les protagonistes peuvent-ils attendre d'une telle confrontation des praxèmes!?

Une des façons d'y répondre est d'envisager les gains respectifs des pilotes dans tous les cas de figure. Excluons d'emblée le premier niveau d'analyse qui ne répond à aucun décodage pertinent. Il reste les tactiques franches - couper à l'intérieur - et les tactiques de ruse - se maintenir ou attaquer à l'extérieur. Afin de pouvoir envisager l'ensemble des possibles de façon rationnelle, il est nécessaire au préalable de fixer quelques règles communes aux pilotes (et conformes à la réalité de la pratique du motocross)!:

Postulats initiaux!:

- La puissance a barre sur la trajectoire!: c'est-à-dire que même moins bien placé au niveau de la piste (excentré du virage, par exemple), le pilote qui possède la moto la 
plus rapide l'emporte s'il est au coude à coude avec un pilote placé à la corde mais moins rapide!;

- La trajectoire a barre sur la position!: de deux pilotes qui se suivent de près, c'est celui qui adopte la trajectoire la plus tendue (moins haut mais plus long lors des sauts, ou à la corde dans les virages) qui passera ou restera en tête.

- Le pilote en tête n'a aucune raison de ménager son effort. Ayant le champ libre, il pilote à vitesse maximale!: nous écrirons désormais vmax pour désigner cet état. Par contre, du fait de l'exiguïté de l'espace individuel d'interaction ou de la gêne du ou des pilotes devançant(s), il est fréquent que les poursuivants ne puissent piloter à leur maximum de vitesse!: nous les désignerons alors!en vsousmax.

Ainsi, la tactique franche du devançant (J1-Sa2) opposée à celle du devancé (J2-Sa2), tout comme la confrontation de tactiques de feinte (J1-Sa3 / J2-Sa3), profitent au devançant qui condamne le poursuivant à emprunter son sillage. Par contre, la ruse de $\mathrm{J} 1$ (J1-Sa3) contre la tactique franche de $\mathrm{J} 2$ (J2-Sa2) donne l'opportunité au devancé de doubler à l'intérieur. Enfin, la tactique franche $(\mathrm{J} 1-\mathrm{Sa} 2)$ contre la ruse $(\mathrm{J} 2-\mathrm{Sa} 3)$ profite au pilote le plus rapide, capable d'exploiter au mieux la puissance de sa machine. On observe fréquemment cette dernière situation quand les leaders sont en passe de prendre un tour aux retardataires. Il est alors sans souci pour eux de doubler à l'extérieur les pilotes dominés qui s'évertuent à couper au plus court dans les virages.

En l'état, la tactique la plus prudente est F (Franc) pour les deux joueurs. Mais elle reste insatisfaisante pour $\mathbf{J} 2$. Le jeu ne présente pas d'équilibre en stratégie pure. Chaque joueur a intérêt à changer de tactique si l'autre maintient la sienne, plus favorable. Il est impossible de prédire à coup sûr la conduite préférentielle des joueurs (cf. Figure 4).

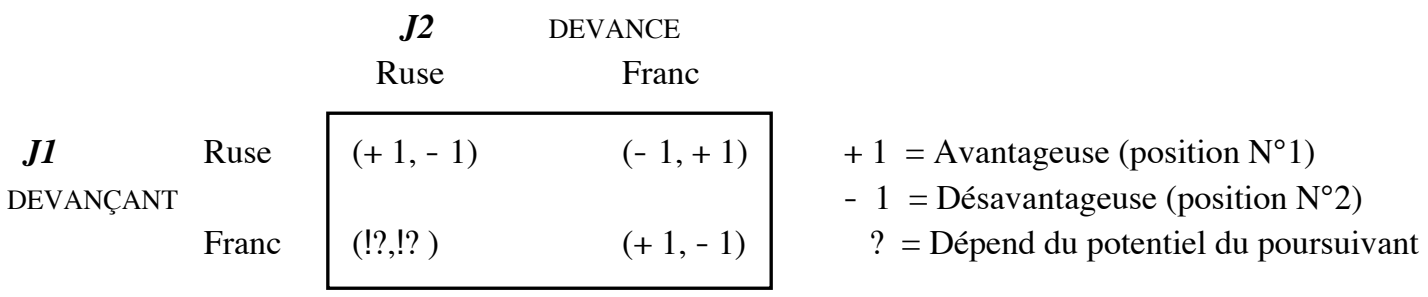

Figure 4. Présentation sous forme normale des scores associés à l'adoption de tactiques franches ou rusées

La tactique du moindre mal invite le devançant et le devancé à jouer Franc (couper la trajectoire). Mais cette situation n'est pas équilibrée. Il n'y a pas de solution en stratégie pure autre que celle dépendant de la connaissance du rapport de force entre les pilotes et leurs machines.

Cependant, le joueur 2 a une information privée. Poursuivant, il est informé de son «!type!», c'est-à-dire de l'état de la situation avant de jouer. Il sait s'il a le potentiel pour doubler à l'extérieur du virage,!J1 maintenant sa cadence (que l'on considère maximale, voir postulat n॰3)!: s'il ne s'en sent pas capable, il se trouve dans l'ETAT 1 (cf. Figure 5)!; s'il s'en sent capable cela revient à l'ETAT 2. Le joueur 1 (devançant) n'est pas informé de l'état mais sait que J2 l'est.

Le joueur 2 va évidemment tenir compte de son information et a ainsi quatre stratégies possibles!: RR (Ruse si ETAT 1, Ruse si ETAT 2), RF (R si ETAT 1, F si ETAT 2...), FR, FF. Par contre, les choix possibles de J1 se réduisent à!: R ou F. Les paiements espérés se calculent aisément (cf. Figure 6). Par exemple, pour (R, RR) on obtient les paiements 
conditionnels $(+1,-1)$ pour l'ETAT 1 , et également $(+1,-1)$ pour l'ETAT 2 . D'où une espérance de gain de $[+1+1] / 2=+1$, pour $\mathrm{J} 1$ et $[-1-1] / 2=-1$, pour $\mathrm{J} 2$.

\begin{tabular}{|c|c|c|c|c|c|}
\hline \multirow{2}{*}{ ETAT 1} & \multicolumn{2}{|c|}{$\boldsymbol{J} \mathbf{2}($ vmax $)$} & \multirow[t]{2}{*}{ ETAT 2} & \multicolumn{2}{|c|}{ J2 (vsousmax) } \\
\hline & Ruse & Franc & & Ruse & Franc \\
\hline Ruse & $(+1,-1)$ & $(-1,+1)$ & Ruse & $(+1,-1)$ & $(-1,+1)$ \\
\hline$J 1$ & & & $J 1$ & & \\
\hline Franc & $(+1,-1)$ & $(+1,-1)$ & Franc & $(-1,+1)$ & $(+1,-1)$ \\
\hline
\end{tabular}

Figure 5. Le jeu est à information incomplète

Le joueur 2 (devancé) a une information privée. Il est informé de son « état 》 (1 ou

2) avant de jouer. Le joueur 1 (devançant) n'est pas informé mais sait que J2 l'est.

À ce petit jeu, $\mathbf{J} 2$ trouve son Minimax (niveau de sécurité) à 0 en choisissant FR. Cela signifie qu'il jouera «!tactique Franche!» (attaquer à l'intérieur du virage) lorsqu'il se sentira incapable de dominer J1 en performance pure (ETAT 1), et «!tactique Ruse!» (attaquer à l'extérieur du virage) lorsqu'il pensera dominer potentiellement J1 (ETAT 2).

Le joueur 1 le sait qui pourra attribuer un ETAT 2 (dominant) à un adversaire devancé qui «!Ruse!» et un ETAT 1 (dominé) à un adversaire se contentant d'attaque «!Franche!».

Mais cette capacité de décentration peut nuire à $\mathrm{J} 1$. La feinte peut opérer au second degré. En effet, J2, bien qu'en ETAT 1 (dominé), peut «!ruser!» (tenter de doubler à l'extérieur) en faisant croire ainsi qu'il est en ETAT 2 (dominant). Car seule la ruse lui est profitable dans ce cas. Se sentant attaqué par un pilote dominateur, le devançant, J1, pare en déployant lui-même la ruse qui devrait, par riposte, maximiser ses gains (il reste à l'extérieur du virage pour empêcher $\mathrm{J} 2$ de passer). Mais en fait, J2 profite de ce bluff et de cette aubaine pour «!jouer franc!» (en coupant la trajectoire) et faire réussir la seule stratégie fructueuse en cas d'infériorité [ETAT 1!; J1 joue R, J2 joue F!; score!: (- 1, $+1)$.

\begin{tabular}{|c|c|c|c|c|}
\hline & & & & \\
\hline & RR & $\mathrm{RF}$ & FR & FF \\
\hline $\mathrm{R}$ & $(+1,-1)$ & $(0,0)$ & $(0,0)$ & $(-1,+1)$ \\
\hline F & $(0,0)$ & $(+1,-1)$ & $(0,0)$ & $(+1,-1)$ \\
\hline
\end{tabular}

Figure 6. Matrice des paiements dans le cas d'une information privée en faveur du pilote devancé (J2)

Le minimum du maximum de satisfaction correspond à la stratégie FR pour J2 (0) et à la tactique $\mathrm{F}$ pour J1 (0). Bien qu'équilibrée la situation est peu satisfaisante pour J1 qui gagnerait à deviner l'état de J2. Mais cette relation empathique n'est pas sans risque.

Le sachant, les pilotes chevronnés qui mènent la course attendent le dernier moment pour réagir. Lorsque le poursuivant jouant $\mathrm{R}$ parvient à arriver à hauteur d'épaule de J1 en prenant une trajectoire plus longue, ce dernier est sûr qu'il n'a pas affaire à un bluff et que l'on est en ETAT 2!; il arrive alors que le moto-cross justifie son appellation, et que $\mathrm{J} 1$ jouant $\mathrm{R}$ in extremis «!bouscule!» $\mathrm{J} 2$ dans les cordes!: la situation est alors momentanément fatale pour l'un des deux protagonistes (ou les deux) qui ira 
(iront) «!au tapis!» (notez qu'elle est définitivement fatale en course motorisée sur circuit où les vitesses dépassent de beaucoup les $50-60 \mathrm{Km} / \mathrm{h}$ du moto-cross). Par contre, ce retard de décision motrice de la part de $\mathrm{J} 1$ peut révéler le bluff de J2 qui n'arrive pas à rattraper le devançant en passant par l'extérieur!; l'information sur le type de $\mathrm{J} 2$ n'est alors plus privée (il est à vmax), et les joueurs jouent l'ETAT 1 au grand dam de J2 qui, démasqué, n'a plus qu'à suivre sagement J1 jusqu'à la ligne d'arrivée pouvant encore miser sur une erreur de pilotage ou une défaillance technique.

D'ailleurs, la monotonie des sports de course que nous avons déjà pu observer [Collard, 1998] tient en grande partie à cet aspect!: sur treize Grands prix automobiles, la probabilité pour les pilotes de passer en tête est de 0,08 alors que celle d'être accidenté est de 0,18!; ce rapport est quasi-identique en moto-cross où l'on double plus souvent mais où l'on chute d'autant plus. Même si l'on ne prend pas en compte le taux d'accidents, il y a fort à parier que des joueurs confrontés à un jeu de société ayant une utilité aussi faible se sépareraient avant même d'avoir commencé. Il y a des intérêts financiers en Formule 1 que nous n'ignorons pas, mais si peu à gagner en moto-cross. C'est peut-être que l'incongruité des relations à trois (ou davantage) laisse présager des occasions inopinées...

\section{APPARITION D'EFFETS PERVERS DANS LES RELATIONS A TROIS}

Nous allons maintenant nous mettre dans une situation à trois joueurs où, théoriquement, l'union peut faire la force. Imaginons que J1 et J'1, deux devançants d'une même équipe soient à la lutte avec J2, le devancé. Possédant des motos de la même écurie, J1 et J'1 caracolent en tête à vmax. Il est un fait que les devançants n'étant gênés par personne sont à même d'exploiter leurs machines au maximum de leurs possibilités!; c'est la raison pour laquelle nous les considérons encore à vmax. Par contre, les premiers ignorent le type de J2. Si J2 est à vmax, l'affrontement prend la forme de l'ETAT 3. Mais comme tout à l'heure, J2 aura pu être gêné au départ et cantonné dans la «meute!» en deçà de son niveau réel. De tour en tour il aura pu refaire son retard et se positionner au moment où nous prenons l'action, en troisième place, à vsousmax, derrière J'1. Si le poursuivant est à vsousmax et que la coalition des devançants est à vmax (ETAT 4) l'opposition ne manque pas de mordant. Comment deux joueurs (vmax) peuvent-ils profiter de leur poste avancé pour contrarier les attaques d'un poursuivant plus rapide (vsousmax)!?

Ainsi que le montre la Figure 7, en ETAT 3, sans aucune entraide particulière, J1 est sûr de maintenir la pole position au sortir du virage en jouant $F$, quoi que fasse J' 1 . En ETAT 4, sans aucune entraide particulière, $\mathrm{J} 1$ et $\mathrm{J}$ '1 ont une chance sur quatre de se faire voler la vedette si J2 Ruse (R) et autant s'il joue Franc (F). En revanche, ils peuvent être sûrs qu'un des deux l'emporte s'ils accordent leurs stratégies. Si J1 joue F et J'1 joue R, J2 est condamné à rester derrière quelle que soit sa stratégie. Idem si J1 joue R alors que J'1 joue F. Par contre, jouer F en simultané ou R ensemble, nuirait à leur réussite collective avec une chance sur deux de concéder la première place à J2.

L'entente profite donc à la paire J1, J'1 en ETAT 4. Mais les sportifs n'ont guère la possibilité de parler. Si nous restons sur notre illustration de moto-cross, ils ne peuvent quasiment pas échanger de «!gestèmes!». En pleine action il leur est impossible de lâcher d'une main leur guidon ni même de tourner franchement la tête!; ils doivent se contenter de hochements de tête, de jambes tendues qui peuvent à la fois désigner une recherche personnelle d'équilibre et l'endroit où aller, etc. Seuls les devancés peuvent 
lire clairement les signaux émis par les devançants. Rappelons qu'en moto-cross, pour des raisons de sécurité, les engins ne possèdent pas de rétroviseur. Ce qui signale au devançant une attaque à l'intérieur ou à l'extérieur, c'est le bruit du moteur de la moto qui s'approche (les casques sont, pour la plupart, munis d'ouïes d'aération à hauteur d'oreilles).

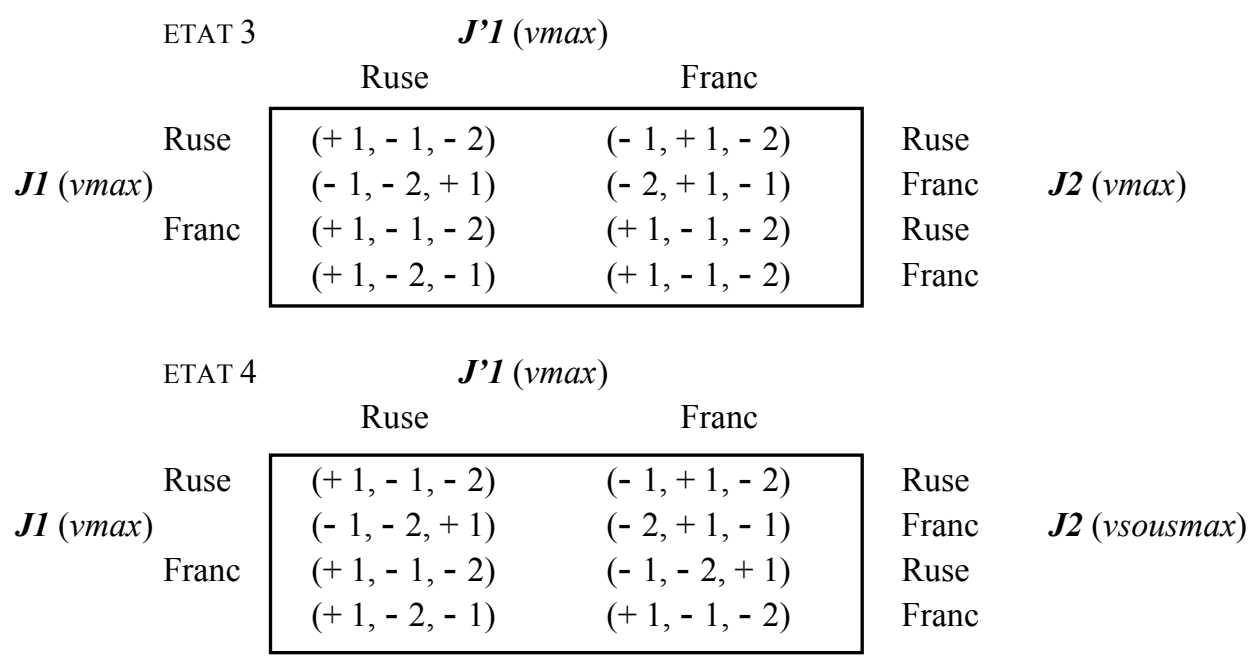

Figure 7. Matrice des gains d'une confrontation à trois joueurs sous forme normale dans le cas d'une information privée en faveur de $\mathrm{J} 2$

Comme précédemment les chiffres entre parenthèses correspondent à la place $(+1$ signifie premier, -1, second, -2, troisième). En ETAT 3, J1 et J'1 se coordonnent en FF, sans avoir besoin de communiquer. En ETAT 4, une coalition est nécessaire pour faire triompher l'équipe 1. Si J1 joue Franc, et J'1 Ruse, J1 reste en tête quoi que fasse J2. Si J1 joue Ruse, et J'1 Franc, J'1 passe en tête quoi que fasse J2. J1 et J'1 vont-ils réussir à s'accorder pour maximiser leur gain ?

Le problème de la résolution de ce jeu n'est donc pas de déterminer ce qu'il y a de mieux à faire pour les équipiers, J1 et J'1 sont d'accord sur ce fait (ou plutôt, les directeurs d'écurie les ont mis «!au parfum!»), mais de s'inquiéter de la transmission du message et de la nature du message qui les fera choisir FF en ETAT 3 et FR plutôt que $\mathrm{RF}$ en ETAT 4. Ces situations sont rarement répétées à l'entraînement, et c'est un tort. J1 et J'1 n'ont pas besoin d'échanger des messages en ETAT 3 et ils le savent!: ils se coordonnent naturellement de façon rentable en FF (cf. Figure 7). L'absence de message entre J1 et J'1 est donc le signe que c'est l'ETAT 3 qui a cours. Cependant, seul J'1 est placé pour percevoir le type de $\mathbf{J} 2$ (avant qu'il ne soit trop tard). Par empathie, ainsi que nous l'avons montré en fin de chapitre précédent, il est à même d'estimer l'état de la confrontation.

Les procédures d'envoi des messages étant libres mais très limitées, la probabilité qu'ils circulent sans défaillance n'est hélas pas égale à un. Chaque message a une probabilité $p !>! 0$ de se perdre ou d'être mal interprété.

- Désignons par S0 le début de la situation!; J'1, en seconde position, réalisant qu'un poursuivant (J2) recolle à leur tandem, estime que l'ETAT 3 ou l'ETAT 4 se profile à partir de la distribution de probabilités $(b, 1-b)$, avec $b>_{-}$(il est en effet plus logique que les poursuivants soient derrière parce qu'ils ne peuvent rouler plus vite);

- $S_{t}$ est le t-ième message envoyé par J'1 si t pair et J1 si t impair!; $I_{t}$ est l'ensemble des informations à t!; 
- IP $=\{\mathrm{J}$ '1 est certain de l'ETAT 3, (J2 reste inexorablement cantonné dans sa roue) et donc n'envoie aucun message à $\mathrm{J} 1$ qui pourrait désigner une entraide \}!;

C'est en effet au devancé de décider en premier de cette possible coopération (il est au courant avant $\mathrm{J} 1$ de la menace représentée par J2) ;

- $\mathrm{I} 0=\{\mathrm{J} 1 \mathrm{n}$ 'a reçu aucun message altruiste $\}$ !;

- $\mathrm{I} 1=\{\mathrm{J} ' 1$, traduit la remontée rapide de $\mathrm{J} 2$ et son agressivité comme la preuve de l'ETAT 4, a envoyé S1 à J2 mais n'a reçu aucune confirmation\}!;

J'1, par exemple, harcelé par J2 dans la ligne droite précédant le virage, utilise un «!praxème!» en se décalant de la trajectoire commune, en se mettant momentanément à hauteur de $\mathrm{J} 1$ avant de se rabattre ostensiblement derrière lui!; il suggère ainsi son intention de se déporter à l'extérieur du virage (R) le moment venu. J1 (qui lui tourne quand même le dos, compte tenu de sa position avancée) l'a-t-il perçu!?

- $\mathrm{I} 2=\{\mathrm{J} 1$ apprend l'ETAT 4 en recevant $\mathrm{S} 1$, envoyé $\mathrm{S} 2$ en retour mais n'a reçu aucune confirmation $\}$ !;

$\mathrm{J} 1$ a bien entendu le vrombissement ostentatoire du moteur de J'1 sur son côté arrière extérieur!qui, s'étant terminé par un rabattement soudain sans que rien ne l'y oblige, est bien le signe de conduites motrices de circonspection ; il est donc averti de la mise en place d'une coopération en ETAT 4. En cette attente, J1 confirme sa trajectoire directe $(\mathrm{F})$ appuyée d'un «!gestème!» comme lever le menton côté extérieur de la piste, là où devrait s'engager J'1 si l'ETAT 4 se confirme. Ce dernier l'a-t-il vu!? Impossible d'en être certain ;

- I3 = \{J'1 a reçu S2, envoyé S3 et n'a pas de confirmation sur ce dernier message $\}$ !; $\mathrm{J}$ '1 a bien perçu l'honorabilité des intentions de $\mathrm{J} 1$ transmises par gestème. Il occupe désormais une position subordonnée, en retrait de $\mathrm{J} 1$ (mais toujours devant $\mathrm{J} 2$ ), destinée à montrer que son action n'est pas un empiètement ou une menace pour la coordination stratégique à venir!; il n'observe aucune réaction supplémentaire chez J1. Le virage se rapproche. Que croit J1 que J'1 pense qu'il fera!? etc.

Dès que $\mathrm{J} 1$ reçoit un message annonciateur de conduites coopératives, $\mathrm{J} 1$ et $\mathrm{J}$ '1 savent séparément qu'ils font en sorte de faire pot commun et qu'on joue l'ETAT 4. Ainsi, à part pour IP et pour I0, l'imprévisibilité ne porte plus sur le pacte ludomoteur, qui devient connu des deux joueurs, mais sur l'état d'information de l'autre joueur. Par exemple en $\mathrm{I} 2$, $\mathrm{J} 1$ a renvoyé un gestème de confirmation $(\mathrm{S} 2)$ et comme il n'a rien vu revenir, il ne sait pas si l'autre joueur est en I1 (J'1 a bien envoyé le premier message mais n'a rien vu revenir) ou en I3 (J'1 a reçu la confirmation S2 et c'est la confirmation S3 qui n'est pas arrivée).

Cette situation, similaire en de nombreux points à celle d'un classique de la théorie des jeux, La bataille des Généraux (deux Généraux devant coordonner l'infanterie et l'artillerie sur la base d'échanges d'informations avec envoi automatique de confirmation dès réception du message, finissent par agir sans concertation à force de douter de l'efficacité de leurs transmissions [Demange, Ponssard, 1994]) implique que quel que soit $p>0$ et quel que soit le nombre de messages échangés, la coordination des stratégies ne peut se faire avec certitude (ceci est renforcé dans notre cas par la polysémie des praxèmes et des gestèmes).

Pour le montrer, calculons les probabilités de $\mathrm{I}_{\mathrm{t}-1}$ et $\mathrm{I}_{\mathrm{t}+1}$ sachant $\mathrm{I}_{\mathrm{t}}$ pour $\mathrm{t} \geq 1$ !:

$$
\begin{aligned}
& \operatorname{prob}\left(\mathrm{I}_{\mathrm{t}-1} \mathrm{I}_{\mathrm{t}}\right)=p /(p+(1-p) p) \\
& \operatorname{prob}\left(\mathrm{I}_{\mathrm{t}+1} \mathrm{I}_{\mathrm{t}}\right)=(1-p) p /(p+(1-p) p)
\end{aligned}
$$

d'où : 


$$
\operatorname{prob}\left(\mathrm{I}_{\mathrm{t}-1} \mathrm{I}_{\mathrm{t}}\right) / \operatorname{prob}\left(\mathrm{I}_{\mathrm{t}+1} \mathrm{I}_{\mathrm{t}}\right)=1 /(1-p)>1
$$

Ainsi, si un joueur a envoyé un message $S_{t}$ et n'a pas reçu de confirmation, il y a plus de chance que $S_{t}$ ait été perdu plutôt que ce soit sa confirmation $S_{t+1}$ qui ne soit pas arrivée.

Par ailleurs, comme nous avons supposé que $b>_{-}$, alors!:

$$
\operatorname{prob}(\mathrm{IP} \backslash \mathrm{I} 0)=b /(b+(1-b) p)>\operatorname{prob}(\mathrm{I} 1 \backslash \mathrm{I} 0)
$$

Donc, si J1 n'a reçu aucun message cohésif, il est plus à même de penser qu'on joue l'ETAT 3 plutôt que le premier message se soit perdu ou ait été mal interprété (une aubaine pour ne pas perdre la face devant les représentants officiels de la tactique commune).

On se retrouve dans une situation particulièrement paradoxale où les joueurs, quel que soit le nombre de messages échangés (messages qui n'ont sens qu'en présence de l'ETAT 4), risquent fort de se coordonner en F (et ainsi obtenir un score commun des plus bas, cf. Figure 8) bien que chacun d'eux, pris isolément, sache avec certitude qu'une coopération était en cours qui devait leur permettre de faire mieux. Finalement dans ces sports à $n$ joueurs et à somme non nulle, la tendance rationnelle spontanée est

\begin{tabular}{|c|c|c|}
\hline \multirow[t]{2}{*}{ ETAT 4} & \multicolumn{2}{|c|}{$\boldsymbol{J} 1$ (vmax) } \\
\hline & Ruse & Franc \\
\hline $\begin{array}{l}\text { Ruse } \\
\boldsymbol{J 1}(\text { vmax })\end{array}$ & $(0,-3 / 2)$ & $(-3 / 2,+1)$ \\
\hline Franc & $(+1,-3 / 2)$ & $(0,-3 / 2)$ \\
\hline
\end{tabular}
aux stratégies motrices «!personnelles!» et ceci au mépris de coopérations fructueuses.

Figure 8. Espérances de gain de J1 et J'1 si J2, en état vsousmax, joue indifféremment $\mathrm{R}$ ou $\mathrm{F}$

La recherche individuelle du « moindre mal » incite $\mathrm{J} 1$ à jouer $\mathrm{F}$ et $\mathrm{J}$ '1 à éviter $\mathrm{R}$. Sans concertation $\mathrm{J} 1$ et J'1 se coordonnent en FF ; alors qu'une entente aboutissant à RF, ou FR permettrait à l'un des deux alliés de rester à coup sûr en tête de la course $(+1)$.

Vérifions ce paradoxe!:

- en I0, J1 n'a pas reçu de message, mais ce qui est sûr c'est qu'il doit jouer F en ETAT 3 tout comme J'1, et F si J'1 joue R et R si J'1 joue F en ETAT 4!: il maximise son espérance de gain sachant qu'il obtiendra!:

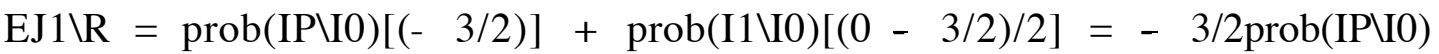
- 3/2prob(I1\0) s'il joue R et, au mieux!:

$\mathrm{EJ} 1 \backslash \mathrm{F}=\operatorname{prob}(\mathrm{IP \backslash} \mathrm{O})[(+1)]+\operatorname{prob}(\mathrm{I} 1 \backslash \mathrm{I} 0)[(+1+0) / 2]=\operatorname{prob}(\mathrm{IP} \backslash \mathrm{I} 0)+1 / 4 \operatorname{prob}(\mathrm{I} 1 \backslash \mathrm{I} 0)$ s'il joue $\mathrm{F}$;

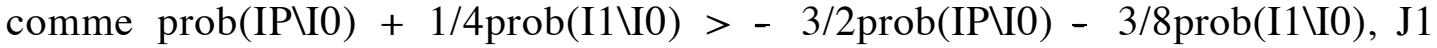
optimisera ses gains conditionnels en jouant $\mathrm{F}$.

- en I1, J'1 sait l'ETAT 4 (coopération engagée) et J1 jouant F!en I0 ; ses espérances de gain conditionnelles sont alors respectivement de!:

EJ' $1 \backslash \mathrm{R}=\operatorname{prob}(\mathrm{I0 \backslash I1})[(-3 / 2)]+\operatorname{prob}(\mathrm{I} 2 \backslash \mathrm{I} 1)[(-3 / 2-3 / 2) / 2]=-3 / 2 \operatorname{prob}(\mathrm{I} 0 \backslash \mathrm{I} 1)$ - 3/2prob(I2\I1) s'il joue R, et!au mieux :

$\mathrm{EJ}^{\prime} 1 \backslash \mathrm{F}=\operatorname{prob}(\mathrm{IO} \backslash \mathrm{I} 1)[(-3 / 2)]+\operatorname{prob}(\mathrm{I} 2 \backslash \mathrm{I} 1)[(+1-3 / 2) / 2]=-3 / 2 \operatorname{prob}(\mathrm{I} 0 \backslash \mathrm{I} 1)$ - 1/4prob(I2\I1) s'il joue F!; 
comme - 1/4prob(I2\I1) > - 3/2prob(I2\I1), J'1 jouera donc également F quel que soit l'état. Par récurrence, les joueurs choisissent toujours F, perdant ainsi l'assurance qu'un des deux maintienne la première place $(+1)$ en jouant la solidarité.

Autrement dit, même si chaque joueur a la certitude qu'un événement se produit (ETAT 4), le fait qu'il ne soit pas certain que les autres le sachent peut exclure des comportements qui tireraient parti de cette certitude [G. Demange, J.-P. Ponssard, 1994, p. 150].

On perçoit ici l'importance de la coordination systématique de praxèmes ou de gestèmes coopératifs à l'entraînement. $\mathrm{Si}$, par exemple, le praxème émis par J'1 (débordement avorté à l'extérieur de J1) était convenu comme étant!«!L'indice!» annonciateur de FR, le «!signal!» au sens de L. Prieto [1966], il ne serait pas nécessaire d'échanger d'autres messages de confirmation et de re-confirmation. D'ailleurs, n'oublions pas que la redondance d'interactions motrices indirectes liant $\mathrm{J} 1$ et $\mathrm{J}$ '1 risque de faire démasquer le complot. L'anticipant, J2 peut très bien tirer bénéfice de la situation en jouant $\mathrm{F}$.

En effet, si $q$ est la probabilité que $\mathrm{J} 1$ joue $\mathrm{F}$ et J'1 joue R (ou l'inverse!: RF), et $(1-q)$ la probabilité que J1 et J'1 jouent FF ou RR, alors les espérances de gain de J2 en ETAT 4 sachant $q>$ _ sont!:

- $\mathrm{EJ} 2 \backslash \mathrm{R}=(1-q) \times(-2+1) / 2+q(-2-2) / 2=-3 / 2 q-{ }_{-}>-5 / 4$ !;

- $\mathrm{EJ} 2 \backslash \mathrm{F}=(1-q) \times(-2+1) / 2+q(-1-1) / 2=-\_q-{ }_{-}>-3 / 4$ !: il vaut donc mieux jouer $\mathrm{F}$ pour $\mathrm{J} 2$ pressentant la coalition entre $\mathrm{J} 1$ et $\mathrm{J}$ '1.

En revanche, par récurrence à rebours, J2 jouera R qui sait qu'en concurrence, J1 et $\mathrm{J}$ '1 se coordonnent à perte en FF. Ainsi, les signaux permettant à des réseaux de coalition d'aboutir à leurs fins doivent être transparents intra- et opaques inter-réseaux.

Un dernier aspect mérite d'être soulevé. Il se peut bien que les dysfonctionnements dans les associations relationnelles positives aient une autre origine que les défaillances du canal et des codes de communication. Dans ces sports à $n$ joueurs et à somme non nulle, les actes de trahison ont pignon sur rue. J1 peut refuser la coopération engagée par J'1, tout simplement parce qu'il doute de la bonne foi de ce dernier. Et si J'1 n'était autre que le serviteur de J2!? Et si J'1 améliorait son sort en servant la cause de J2!? Nous avons jusqu'ici envisagé les connivences des devançants!; voici celles des devancés.

J'1 - que nous désignerons à présent J'2 pour signifier son caractère ambivalent ne peut jouer de façon satisfaisante avec J1 sans un pacte préalable, nous venons de le voir. Par contre, une association fortuite entre lui (J'2) et $\mathrm{J} 2$ peut s'avérer à coup sûr plus fructueuse. En effet, dans l'expectative sur ce que jouera J1 en ETAT 4, J'2 et J2 vont trouver naturellement un équilibre en jouant FR (cf. Figure 9)!; avec, à la clef pour J'2, un Maximin - c'est-à-dire un maximum du minimum de satisfaction possible (le mieux qu'il puisse obtenir si le pire arrive) - meilleur que s'il pactisait avec J1.

Si $\mathrm{J} 1$ joue avec une équiprobabilité $\mathrm{F}$ et $\mathrm{R}, \mathrm{J}$ '2 associé à $\mathrm{J} 2$ obtient son Maximin à - _. en jouant $\mathrm{F}$; alors que si J2 joue avec une équiprobabilité $\mathrm{F}$ et $\mathrm{R}, \mathrm{J}$ '2 (nommé alors $\mathrm{J}$ '1) associé à $\mathrm{J} 1$ obtient son Maximin à - 3/2 également en jouant $\mathrm{F}$. Pour J'2, mieux vaut jouer $\mathrm{F}$ contre $\mathrm{J} 1$ avec $\mathrm{J} 2$, que jouer $\mathrm{F}$ contre $\mathrm{J} 2$ avec $\mathrm{J} 1$. 


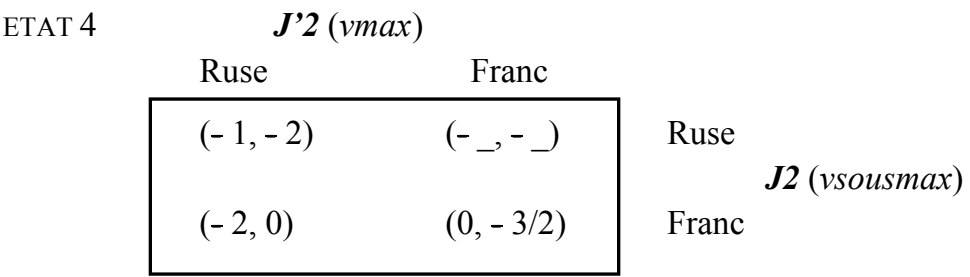

Figure 9. Espérances de gain de $\mathrm{J} 2$ et J'2 si J1 joue indifféremment $\mathrm{R}$ ou $\mathrm{F}$

Sans besoin de concertation J'1, alias J'2, et J2 se coordonnent en équilibre avec FR, leur Minimax : aucun des deux n'a intérêt à changer de tactique si l'autre maintient la sienne. Dans cette situation, J'2 joue également un Maximin (- _) plus satisfaisant que lorsqu'il envisageait sa coopération avec l'autre membre de son équipe, J1 (-3/2). Dans ce type de jeu mieux vaut parfois « pactiser avec le diable ».

Dans ce type d'interaction à trois (en ETAT 4), le pilote intermédiaire a le sort de la partie entre ses mains. Sur le plan stratégique, c'est l'élément énigmatique. Il porte en lui tout ce qui distingue ces sports de course de ceux à information complète ou parfaite. Il est le vecteur du piment du jeu. Si Doctor Jekyll (alias J'1) est disposé à jouer de concert avec $\mathrm{J} 1$, il a la possibilité - si et seulement si canal et code de communication sont maîtrisés - de faire gagner son équipe à tous les coups et durablement avec une probabilité de _ (car seule $(+1,-1,-2)$ est une solution durable pour les deux devançants, que l'équipe obtient avec $d={ }_{-}$si J'1, J1 jouent FR ou RF) et ce, quoi que fasse J2. Mais, s'il se transforme en Mister Hyde (alias J'2) en s'acoquinant inopinément avec $\mathbf{J} 2$, il est certain que l'un des deux prendra le commandement de la course quoi que fasse J1. Si le profit escompté pour Hyde est meilleur dans l'absolu (qu'en jouant Jekyll), il ne l'est que de courte durée... En effet, ayant pris la tête de la course, il suffit aux deux nouveaux poursuivants de laisser libre cours à leur insatisfaction (pour J2) et à leur esprit de vengeance (pour J1) pour refaire à J'2 ce qu'il vient de faire à J1. Dans ce cercle vicieux de trahisons successives, le grand bénéficiaire reste $\mathrm{J} 2$. Il lui suffira en effet de valider une seule fois la première place pour la garder jusqu'à l'arrivée (en ETAT 4, il est le seul à être à vsousmax).

À présent on perçoit le caractère fragile des interactions indirectes dans le cas des sports à somme non nulle où $n>2$. Même si les codes de communication sont transmis à bon escient et reconnus - ce qui n'est déjà pas donné en moto-cross comme nous l'avons vu - les coalitions officielles peuvent être délibérément évitées car elles sont subordonnées aux coalitions de fortune!; et des adversaires peuvent conjointement tirer parti d'une situation problématique en devenant momentanément partenaires. Cette ambivalence est violemment combattue en cyclisme ou en Formule 1 où les enjeux financiers et médiatiques exercent un véritable dictat sur les conduites motrices à tenir. Les pré-décisions «!rationnelles!» doivent dans ce cas s'appliquer ipso facto sur le champ de jeu sous peine de sanction voire d'exclusion. Des joueurs se savent par avance sacrifiés au bénéfice du leader reconnu!; et ils l'acceptent au mépris de la logique interactionnelle propre à cette espèce de jeu. Dans ce cas, une structure de coalition d'équipes supplante la structure initiale de coalition de joueurs. Mais rien, dans la logique interne du jeu, n'oblige les pilotes!; contrairement à ce qui se passe en football ou en rugby où de telles interrogations sur la nécessité ou non d'adhérer à la politique d'équipe sont exclues. 


\section{COMMUNICATION PRAXIQUE MANQUEE}

Si l'on s'en tient au schéma élémentaire de la communication de Cl. Shannon [1975], pour communiquer il faut!toujours : un émetteur, un récepteur, un canal par lequel s'acheminent les messages, eux-mêmes soumis aux contraintes d'un code. En motocross, si l'opacité des messages participe de l'ambivalence relationnelle, qu'en est-il de la maîtrise du système de règles!porteur de ces messages ? Les pilotes de moto-cross parlent-ils la même langue!?

Pour le savoir, nous avons interrogé une cinquantaine de pilotes émérites (de niveau de compétition nationale) participant pour la plupart à l'enduro du Touquet de février 2003. À la question!: Durant les compétitions, vous arrive-t-il de coopérer avec d'autres sportifs en cours d'épreuve?, la réponse majoritaire est affirmative (cf. Figure 10). On observe une tendance équivalente (même renforcée) chez 25 étudiants sportifs spécialistes de cyclisme. À vrai dire ces réponses marquées ne laissent pas de surprendre car de telles interactions cohésives sont quasi imperceptibles lors de leur accomplissement, surtout en motocyclisme. Sans doute est-ce le fruit d'un savoir-faire particulier fondé sur l'encodage et le décodage d'indices comportementaux.

\begin{tabular}{|c|c|c|c|c|}
\hline $\begin{array}{c}\text { Coopérez-vous } \\
\text { souvent } ?\end{array}$ & Jamais & Rarement & Souvent & Toujours \\
\hline 53 motards & $7 \%(4)$ & $23 \%(12)$ & $49 \%(26)$ & $21 \%(11)$ \\
\hline 25 cyclistes & 0 & $12 \%(3)$ & $68 \%(17)$ & $20 \%(5)$ \\
\hline
\end{tabular}

Figure 10. Fréquence de coopérations interpersonnelles chez des experts de moto-cross et de cyclisme

La tendance est à la coopération ! Si cela est conforme à l'idée que l'on peut se faire de coalitions

d'équipes (cyclisme), par contre on s'en étonne s'agissant de coalitions d'individus (moto-cross).

On pouvait pourtant légitimement s'attendre à ce que les trahisons potentielles minent la générosité des pilotes et qu'ils finissent d'ailleurs par jouer «!chacun pour soi!» (eu égard aux résultats du chapitre précédent). Ce n'est pas fondamentalement le cas!: ils disent plus souvent coopérer que s'abstenir de le faire. Pour autant, ils nuancent un peu plus tard dans le questionnaire lorsqu'ils présentent «!les trahisons!» comme premier facteur pouvant empêcher les coopérations, devant «!la difficulté de se mettre d'accord!» et le fait «!qu'on ne s'entraîne jamais dans ce sens!»... Dans de tels sports, comment peut-on communiquer si le langage pose problème et qu'aucun apprentissage spécifique ne lui est consacré!?

Toutefois, le biais introduit par ce type de question directe est désormais bien connu en sociologie [J-P. Grémy, 1992]!: les répondants ne sont pas de simples répondeurs adeptes du stimulus-réponse et leurs propos représentent plutôt l'image qu'ils souhaitent donner pour être conforme à la problématique suggérée. Une chose est donc d'avancer une réponse en raison de l'effet qu'elle produit, une autre est de savoir ce qu'elle signifie. Puisque les motards semblent sûrs de leur fait, comment font-ils!pour communiquer?

De quelles façons communiquez-vous lors de vos actes de coopération? [leur demande-t-on]

Nous vous proposons cinq façons de transmettre des messages de coopération : 
- Par l'action même que vous engagez comme se décaler à l'extérieur, s'engager vers le centre, pour donner une indication, demander quelque chose, etc. (codé A pour «!Action»),

- Par des cris ou sifflements pour donner une indication, demander quelque chose, etc. (codé C pour «!Cri!»),

- Par gestes des membres comme tendre la jambe libre, montrer du doigt; ou de la tête, la lever, la tourner, pour donner une indication, demander quelque chose, etc. (codé G pour «!Geste!»),

- Par des mots, une phrase pour donner une indication, demander quelque chose, etc. (codé M pour «!Mot!»),

- Par une posture du corps particulière comme s'avancer sur le réservoir, se mettre en appui sur ses repose-pieds pour donner une indication, demander quelque chose, etc. (codé P pour «!Posture!»).

... merci de bien vouloir les classer par ordre d'importance, du plus vers le moins...

On notera que ces cinq critères se veulent exhaustifs!; 1'un correspond à la classe des praxèmes c'est-à-dire aux actions motrices qui portent le projet tactique (A)!; la posture du corps $(\mathrm{P})$, l'organisation du pilote sur sa moto, n'est pas un praxème à part entière mais un indice majeur susceptible de rentrer dans sa constitution!; les mouvements des membres $(\mathrm{G})$ représentent la classe des gestèmes, ils ne sont pas constitutifs de la situation motrice elle-même mais s'y surajoutent tout comme les cris ou sifflements (C) qui, avec les mots (M) renvoient au code classique langagier. Retenir des phrases ou des mots comme moyen de communication d'un sport qui brasse des dizaines de décibels peut sembler inopportun. Toutefois, dans les épreuves d'enduro (dont une majorité de répondants est spécialiste) les arrêts pour ravitaillement ou orientation sont présents ce qui autorise l'emploi de ce système de règles.

Le classement majoritaire donne!:

$\mathrm{A}>\mathrm{P}>\mathrm{G}>\mathrm{C}>\mathrm{M}$ où «! $>$ !» signifie «!plus utilisé pour communiquer que!» (pour simplifier, nous écrirons désormais à la suite APGCM).

Il apparaît que les praxèmes $(\mathrm{A})$ ou leurs modalités $(\mathrm{P})$ sont privilégiés sur les gestèmes $(\mathrm{G})$ eux-mêmes plus fréquemment utilisés que les codes langagiers $(C$ et $M)$. Ce résultat est logique tant il est vrai que parler ou s'agiter donne plus facilement d'information à ceux dont on ne doit pas éveiller les soupçons (les concurrents). Les praxèmes eux, pour être compris, nécessitent une véritable maîtrise sémiologique, ils sont «!moins spontanément visibles!»!: ce qui permet aux émetteurs d'avoir plus de chance de ne rencontrer que les récepteurs légitimes (les coéquipiers).

Sur les $5 !=120$ ordinations possibles de cinq éléments, 23 classements différents apparaissent chez nos 53 répondants. C'est peu au regard du maximum possible $(23 / 120=19 \%)$ mais beaucoup, rapporté à l'effectif étudié $(23 / 53=43 \%)$ !: il y a presque une chance sur deux qu'un tournoi se présente une fois et une seule chez nos 53 motards. L'ordre majoritaire n'est donc nullement ici un indicateur d'homogénéité groupale. D'ailleurs, pour obtenir le résultat à la pluralité nous avons attribué, pour chaque élément de chaque classement individuel, un nombre de points!: 5 pour le critère placé en première place, 4 pour le second, 3 pour le troisième... Au final, il ressort 196 points pour $A, 183$ pour $\mathrm{P}, 177$ pour $\mathrm{G}, 155$ pour $\mathrm{C}$ et 84 pour $\mathrm{M}$. Les quatre premiers critères se tiennent dans un mouchoir de poche et laissent présager des logiques individuelles bigarrées. N'est-ce pas surprenant!? C'est comme si à la question!: Quelle langue est la plus parlée dans votre ville? Classez par ordre décroissant entre, le 
français, l'anglais, l'espagnol, l'allemand et le russe... on obtenait quatre langues sur cinq au coude à coude. On en viendrait à douter que les répondants sont issus de la même ville ou qu'ils communiquent vraiment entre eux. Même dans une ville polyglotte, il est probable que les classements individuels tendent à se calquer sur la réponse majoritaire, par connaissance du milieu. Or c'est ici que le bât blesse chez nos motards. Ont-ils une bonne connaissance des codes de communication qu'ils disent «!souvent» et parfois «!toujours » utiliser!? Derrière le discours de bonnes intentions n’y a-t-il pas un aveuglement relationnel manifeste!?

Pour le savoir nous pouvons utiliser la procédure de Black [G. Guilbaud, 1968]. Si nous considérons le tournoi majoritaire comme étant l'ordre de Black latent, on peut repérer parmi les 53 réponses, celles qui sont blackiennes, c'est-à-dire qui relèvent d'une même cohérence groupale. On peut à partir d'un classement de $n$ éléments dénombrer $2^{n-1}$ ordres homogènes par rapport à cet ordre latent. Pour $n=5$, il vient 16 ordinations blackiennes, c'est-à-dire homogènes par rapport au classement général : APGCM. Pour les trouver, il suffit, une fois désignée la meilleure façon de communiquer, de toujours respecter la proximité relative des quatre autres par rapport à l'ordre latent. Soit : G, le code jugé le plus favorable pour communiquer. Pour être blackiens, les codes suivants seront alors obligatoirement rangés par ce votant selon leur proximité relative vis-à-vis de G dans l'ordre latent. Les deux ordinations GPA et GCM seront toujours respectées. Les différents rangements blackiens réalisables seront dus à toutes les interpolations possibles entre ces deux groupes de codes de communications dont l'ordre interne, qui se déploie de part et d'autre de G, sera toujours reproduit. Ainsi, les classements GPACM ou GCMPA ou encore GPCMA sont des ordres blackiens visà-vis de l'ordre de base, symptomatiques, d'une homogénéité groupale. Par contre, les classements GAPCM ou GACPM ne sont pas blackiens - ils ne respectent pas l'ordre de proximité relative de chaque critère vis-à-vis des autres dans le tournoi latent.

\begin{tabular}{|c|c|c|}
\hline & Ordres blackiens & $\begin{array}{c}\text { Fréquence } \\
\text { d'apparition }\end{array}$ \\
\hline 1 & APGCM & $6 \mathrm{x}$ \\
2 & PAGCM & $4 \mathrm{x}$ \\
3 & PGACM & $1 \mathrm{x}$ \\
4 & PGCAM & $0 \mathrm{x}$ \\
5 & PGCMA & $0 \mathrm{x}$ \\
6 & GPACM & $2 \mathrm{x}$ \\
7 & GPCAM & $0 \mathrm{x}$ \\
8 & GPCMA & $0 \mathrm{x}$ \\
9 & GCMPA & $0 \mathrm{x}$ \\
10 & GCPMA & $0 \mathrm{x}$ \\
11 & GCPAM & $0 \mathrm{x}$ \\
12 & CMGPA & $0 \mathrm{x}$ \\
13 & CGMPA & $0 \mathrm{x}$ \\
14 & CGPMA & $0 \mathrm{x}$ \\
15 & CGPAM & $1 \mathrm{x}$ \\
16 & MCGPA & $0 \mathrm{x}$ \\
\cline { 2 - 3 } & TOTAL $/ 53$ & $14(26 \%)$ \\
\hline
\end{tabular}

Figure 11. Fréquence d'apparition de tournois blackiens sur 53 réponses de motards L'ordre de Black latent (APGCM) fait naître 16 ordinations blackiennes distinctes : cinq d'entre-elles sont choisies par $26 \%$ des motards. En majorité, ce public n'adhère pas à la cohérence de Black. 
Ci-dessus (Figure 11), nous présentons les 16 ordres blackiens eu égard à l'ordre latent APGCM et la fréquence avec laquelle ils ont été cités par les 53 sportifs interrogés. Ce résultat tend à renforcer l'impression première!: seulement un quart $(14 / 53)$ des répondants sont blackiens ce qui ne contredit pas l'hypothèse d'une hétérogénéité groupale. Certes, les motards disent communiquer!; mais ils n'utilisent pas la même langue.

Une autre façon d'observer la distance entre les appréciations individuelles et ce qu'il en transparaît en collectif est d'utiliser le coefficient de Kendall. Il s'agit d'une corrélation de rang obtenu par $\mathrm{K}=1-2 d / \mathrm{D}$ où $d$ est le nombre de désaccords entre deux échelles (en l'occurrence ici entre chaque échelle individuelle et l'échelle collective, cf. Figure 12) et $\mathrm{D}=n(n-1) / 2$ la distance maximale entre deux échelles (avec $n=5, \mathrm{D}=$ 10). $\mathrm{K}$ oscille entre -1 (corrélation maxi- négative) et +1 (corrélation maxi- positive). Entre - _ et $+_{\ldots}$, on peut dire que la corrélation est non significative. Or c'est le cas dans les deux tiers des cas!: à 35 reprises sur 53 tournois individuels le Kendall reste dans cette tranche!; et il n'y a que six classements qui reprennent l'ordre majoritaire à l'identique $(11 \%)$. L'hypothèse émise à partir de l'ordre de Black se confirme. Notre sous-groupe de motards communique peut-être avec constance!; mais ils le font chacun avec leurs codes privilégiés, ce qui risque inévitablement de générer quelques ratés. D'ailleurs, il est un fait qu'à l'entraînement on ne répète jamais de combinaisons tactiques comme au football ou au rugby.

La sémiologie de la motricité dont on a pu, dans le point précédent, repérer le rôle-clef semble bien éloignée des usages motocyclistes, centrés depuis toujours - et comme dans beaucoup de sports - sur la technique pour la technique. Bien que sport sociomoteur à part entière, le moto-cross est envisagé par ses usagers comme un sport psychomoteur où les interrelations apparaissent évidentes tout en ne nécessitant aucune prise en compte effective.

\begin{tabular}{|c|c|c|c|}
\hline $\begin{array}{c}\text { Classements } \\
\text { Individuels }\end{array}$ & Effectif & $\mathrm{d}$ & $\mathrm{K}$ \\
\hline APGCM & $6 \mathrm{x}$ & 0 & $\mathbf{+ 1}$ \\
\hline PCGAM & $5 \mathrm{x}$ & 4 & $+0,2$ \\
\hline GCAPM & $5 \mathrm{x}$ & 4 & $+0,2$ \\
\hline PAGCM & $4 \mathrm{x}$ & 1 & $+\mathbf{0 , 8}$ \\
\hline PCMAG & $4 \mathrm{x}$ & 5 & 0 \\
\hline AGPMC & $3 \mathrm{x}$ & 2 & $+\mathbf{0 , 6}$ \\
\hline AMGCP & $3 \mathrm{x}$ & 5 & 0 \\
\hline APMCG & $3 \mathrm{x}$ & 3 & $+0,4$ \\
\hline GPACM & $2 \mathrm{x}$ & 3 & $+0,4$ \\
\hline GACPM & $2 \mathrm{x}$ & 3 & $+0,4$ \\
\hline AGCPM & $2 \mathrm{x}$ & 2 & $\mathbf{+ 0 , 6}$ \\
\hline GAPCM & $2 \mathrm{x}$ & 2 & $\mathbf{+ 0 , 6}$ \\
\hline
\end{tabular}

\begin{tabular}{|c|c|c|c|}
\hline $\begin{array}{c}\text { Classements } \\
\text { individuels }\end{array}$ & Effectif & $\mathrm{d}$ & $\mathrm{K}$ \\
\hline CPGAM & $2 \mathrm{x}$ & 5 & 0 \\
\hline PGACM & $1 \mathrm{x}$ & 2 & $+\mathbf{0 , 6}$ \\
\hline CGPAM & $1 \mathrm{x}$ & 6 & $-0,2$ \\
\hline PCAMG & $1 \mathrm{x}$ & 4 & $+0,2$ \\
\hline ACGPM & $1 \mathrm{x}$ & 3 & $+0,4$ \\
\hline CGAPM & $1 \mathrm{x}$ & 5 & 0 \\
\hline CAGPM & $1 \mathrm{x}$ & 4 & $+0,2$ \\
\hline PGAMC & $1 \mathrm{x}$ & 3 & $+0,4$ \\
\hline GPAMC & $1 \mathrm{x}$ & 4 & $+0,2$ \\
\hline ACGMP & $1 \mathrm{x}$ & 4 & $+0,2$ \\
\hline AGCMP & $1 \mathrm{x}$ & 3 & $+0,4$ \\
\hline $23 \mathrm{x}$ & $53 \mathrm{x}$ & &
\end{tabular}

Figure 12. Ensemble des tournois individuels, effectif, distance et corrélation (K) vis-à-vis de l'ordre majoritaire (APGCM)

En gras dans le tableau apparaissent les classements individuels en corrélation significative positive avec le classement à la pluralité des voix : ils ne représentent que 18 des 53 tournois (34\%). Le sous-groupe de motards interrogé est composite. 


\section{CONCLUSION. UNE FORMULE EN DÉCLIN!?}

Les sports de course avec structures de coalitions de joueurs possèdent un potentiel interactionnel mésestimé - y compris par les joueurs eux-mêmes qui se contentent, comme on l'a vu, d'un discours de bonnes intentions à propos de la communication.

La théorie des jeux, souvent décrite comme un outil stérile et un simple enjoliveur de données, permet de mettre au jour l'impérieuse nécessité d'avancer dans le domaine de la sémiologie de la motricité, du moins pour ces sports à $n$ joueurs et à somme non nulle. En effet, faute d'un accord préalable sur un code praxique spécifique, on montre que les joueurs finissent par renoncer à des coopérations fructueuses en jouant à perte chacun pour soi. À moins de subir les pressions d'une écurie ou d'un «!team!», les trahisons ne pourront être empêchées pour autant (même si - dans notre exemple - nous avons vu qu'il ne pouvait s'agir que de profits supplémentaires passagers).

C'est ce caractère relationnel équivoque qui rend sans doute ces sports les plus enthousiasmants. Face à la pureté des duels et à leur exclusivité draconienne, face à la simplicité des sports psychomoteurs et à leur sens pratique de l'équité, ils détonnent et rappellent aussi que la vie sociale n'est pas faite que de rapports dichotomiques simplistes à information complète voire parfaite.

Mais l'Institution sportive n'entend pas gâcher le spectacle. Le sport se construit pour les médias et les praxèmes sont invisibles à l'œil nu des profanes. Aussi, la formule des courses est-elle retravaillée sans cesse dans sa chair (et dans ce qu'elle a de plus cher). Et l'on organise désormais des concours de saut au cas par cas en moto-cross dans les arènes de Paris-Bercy. Et l'on modifie - prétextant de légitimes problèmes de sécurité - le tracé des circuits automobiles afin que la résolution du jeu se fasse si possible la veille des Grands prix, sans interaction, durant les essais...

\section{BIBLIOGRAPHIE}

COLLARD L., Sports, enjeux et accidents, Paris, Presses Universitaires de France, 1998.

DEMANGE G., PONSSARD, J-P., Théorie des jeux et analyse économique, Paris, Presses Universitaires de France, 1994.

GRÉMY J-P., «!La formulation des questions d'enquête!: son effet sur les réponses!», La qualité de l'information dans les enquêtes, Paris, Dunod, 1992, p. 97-114.

GUILBAUD G., Eléments de la théorie mathématique des jeux, Paris, Dunod, 1968.

PARLEBAS P., «!Une rupture culturelle!: des jeux traditionnels au sport!», Le sport à corps et à cris, Revue internationale de psychosociologie, $\mathrm{n}^{\circ} 20$, Paris, Eska, 2003, p. 9-36.

PARLEBAS P., Jeux, sports et sociétés. Lexique de praxéologie motrice, Paris, Institut National du Sport et de l'Education Physique, 1999.

PARLEBAS P., «!Réseaux dans les jeux et les sports!», L'année sociologique, vol. 52 / n'2, 2002, p. 315-349.

POPOV A., Nager dans le vrai, Paris, Le cherche midi éditeur, 2001.

PRIETO L., Messages et signaux, Paris, Presses Universitaires de France, 1966.

SHANNON C., Théorie mathématique de la communication, Paris, Retz, CEPL, 1975.

VON NEUMANN J., MORGENSTERN O., Theory of games and economic behavior, Princeton, University Press, 1944. 